\title{
Muon diffusion and electronic magnetism in $\mathrm{Y}_{2} \mathrm{Ti}_{2} \mathrm{O}_{7}$
}

\author{
J. A. Rodriguez, ${ }^{1, *}$ A. Yaouanc, ${ }^{1,2}$ B. Barbara ${ }^{3}$ E. Pomjakushina, ${ }^{4}$ P. Quémerais, ${ }^{3}$ and Z. Salman ${ }^{1, \dagger}$ \\ ${ }^{1}$ Laboratory for Muon-Spin Spectroscopy, Paul Scherrer Institut, CH-5232 Villigen-PSI, Switzerland \\ ${ }^{2}$ Institut Nanosciences et Cryogénie, SPSMS, CEA and University Joseph Fourier, F-38054 Grenoble, France \\ ${ }^{3}$ Institut Néel, CNRS and Université Joseph Fourier, B.P. 166, 38042 Grenoble, Cedex 09, France \\ ${ }^{4}$ Laboratory for Developments and Methods, Paul Scherrer Institut, CH-5232 Villigen-PSI, Switzerland \\ (Received 11 January 2013; revised manuscript received 29 April 2013; published 24 May 2013)
}

\begin{abstract}
We report a muon spin relaxation study in a $\mathrm{Y}_{2} \mathrm{Ti}_{2} \mathrm{O}_{7}$ single crystal. We observe slow local field fluctuations at low temperature which become faster as the temperature is increased. Our analysis suggests that muon diffusion is present in this system and it is small below $40 \mathrm{~K}$ and therefore incoherent. A surprisingly strong electronic magnetic signal is observed with features typical for muons thermally diffusing towards magnetic traps below $\approx 100 \mathrm{~K}$ and released from them above this temperature. We attribute the traps to $\mathrm{Ti}^{3+}$ defects in the diluted limit. Our observations are highly relevant to the persistent spin dynamics debate on $R_{2} \mathrm{Ti}_{2} \mathrm{O}_{7}$ pyrochlores and their crystal quality.
\end{abstract}

DOI: 10.1103/PhysRevB.87.184427

PACS number(s): 66.30.-h, 75.10.Jm, 61.72.-y, 76.75.+i

\section{INTRODUCTION}

The rare-earth titanates and stanates series of compounds, $R_{2} M_{2} \mathrm{O}_{7}$ ( $R$ is a rare-earth ion and $M$ is Ti or $\mathrm{Sn}$ ), which crystallize in the pyrochlore crystal structure (space group $F d \overline{3} m$ ), are prone to strong geometrical frustration. ${ }^{1}$ Their study has revealed a wealth of exotic magnetic properties. These include (i) the spin-ice ground state of $\mathrm{Ho}_{2} \mathrm{Ti}_{2} \mathrm{O}_{7}$ and $\mathrm{Dy}_{2} \mathrm{Ti}_{2} \mathrm{O}_{7},{ }^{2-4}$ (ii) the ground state reached by $\mathrm{Yb}_{2} \mathrm{Ti}_{2} \mathrm{O}_{7}$ after a sharp transition in the spin dynamics fingerprinted by a pronounced peak in the specific heat, ${ }^{5}$ (iii) the unconventional dynamical ground state of $\mathrm{Tb}_{2} \mathrm{Sn}_{2} \mathrm{O}_{7}$ for which magnetic Bragg reflections are observed by neutron diffraction, ${ }^{6}$ while no spontaneous magnetic field is measured by the zero field (ZF) muon spin relaxation ( $\mu \mathrm{SR}$ ) technique, ${ }^{7}$ (iv) the persistent spin dynamics (PSD) detected in the ordered states of $\mathrm{Gd}_{2} \mathrm{Sn}_{2} \mathrm{O}_{7}, \mathrm{Gd}_{2} \mathrm{Ti}_{2} \mathrm{O}_{7}$, and $\mathrm{Er}_{2} \mathrm{Ti}_{2} \mathrm{O}_{7},{ }^{8-12}$ and the nonordered PSD state of $\mathrm{Tb}_{2} \mathrm{Ti}_{2} \mathrm{O}_{7} \cdot 13,14$

The first report of PSD was for $\mathrm{SrCr}_{8} \mathrm{Ga}_{4} \mathrm{O}_{19}$ using $\mu \mathrm{SR} .{ }^{15}$ It was found that this kagome compound does not exhibit magnetic Bragg reflections down to $50 \mathrm{mK}$. Although mostly observed by $\mu \mathrm{SR}$, PSD has been also proposed by other techniques, e.g., in $\mathrm{Gd}_{2} \mathrm{Sn}_{2} \mathrm{O}_{7}$ using ${ }^{155} \mathrm{Gd}$ Mössbauer spectroscopy. ${ }^{8}$ Both $\mu \mathrm{SR}$ and the neutron spin echo have observed PSD in $\mathrm{Tb}_{2} \mathrm{Sn}_{2} \mathrm{O}_{7}{ }^{7,16}$ as well as in $\mathrm{Dy}_{2} \mathrm{Ti}_{2} \mathrm{O}_{7}{ }^{4,17,18}$ However, the existence and nature of PSD is still under debate. ${ }^{9,19}$ Recently, it was suggested that PSD does not exist in $\mathrm{Dy}_{2} \mathrm{Ti}_{2} \mathrm{O}_{7}$ and that the observed relaxation results from coherent muon diffusion. ${ }^{20}$ In that work it was proposed to perform $\mu$ SR measurements in $\mathrm{Y}_{2} \mathrm{Ti}_{2} \mathrm{O}_{7}$ to investigate the possibility of coherent muon diffusion in a compound where frustrated magnetism does not play any role as no $4 f$ magnetism should be present. In this paper we report $\mathrm{ZF}$ and longitudinal field (LF) $\mu$ SR measurements on $\mathrm{Y}_{2} \mathrm{Ti}_{2} \mathrm{O}_{7}$. We show that there is no detectable neutral muonium formation, and that the positive-muon hopping rate at low temperature is much smaller than what was proposed for $\mathrm{Dy}_{2} \mathrm{Ti}_{2} \mathrm{O}_{7} \cdot{ }^{20}$ Also, we have observed a relatively strong muon spin relaxation which we attribute to a small density of $\mathrm{Ti}^{3+}$ magnetic defects. Note that this system has already been studied by Dunsiger using $\mu \mathrm{SR} .{ }^{21}$ Nevertheless, that study was less extensive than the one reported here and did not consider the possibility of coherent diffusion at low temperature. When a comparison is possible, our data and that from Dunsiger are similar.

\section{EXPERIMENT}

Polycrystalline $\mathrm{Y}_{2} \mathrm{Ti}_{2} \mathrm{O}_{7}$ was prepared by a solid state reaction. Starting materials of $\mathrm{Y}_{2} \mathrm{O}_{3}$ and $\mathrm{TiO}_{2}$ with $99.99 \%$ purity were mixed and ground. Then they were heat treated at $900-1150^{\circ} \mathrm{C}$ in air for more than $100 \mathrm{~h}$ with several intermediate grindings. The resulting powder was hydrostatically pressed in the form of rods $(8 \mathrm{~mm}$ in diameter and $60 \mathrm{~mm}$ in length). The rods were subsequently sintered at $1150{ }^{\circ} \mathrm{C}$ during $15 \mathrm{~h}$. A single crystal was grown using an optical floating zone furnace with four $1000 \mathrm{~W}$ halogen lamps as a heat source. The growth was done in 4 bar pressure of an argon and oxygen mixture (50:50), at a growth rate of $10 \mathrm{~mm} / \mathrm{h}$, and with the feeding and seeding rods rotated at $20 \mathrm{rpm}$ in opposite directions (to maintain homogeneity of the liquid). The crystal was postannealed at $1150^{\circ} \mathrm{C}$ in argon for $15 \mathrm{~h}$ in order to remove possible overstoichiometric oxygen. Phase purity of the grown crystal was checked using conventional $\mathrm{x}$-ray diffraction and the obtained lattice parameter $a=10.099 \AA$ is in good agreement with the literature. ${ }^{22}$ The crystal was then aligned using an $\mathrm{x}$-ray Laue camera, and magnetization measurements were performed down to $1.9 \mathrm{~K}$ (see Fig. 1).

The $\mu \mathrm{SR}$ measurements were carried out in the general purpose spectrometer at the Swiss Muon Source facility of the Paul Scherrer Institut (Switzerland). Most of the measurements were done in the LF geometry for which the initial muon spin and the external magnetic field $\mathbf{B}_{\text {ext }}$ are parallel. ${ }^{23,24}$ We define the $Z$ axis to be parallel to the initial muon spin direction, with the positron detectors centered along this axis. With this geometry the measured asymmetry (i.e., $\mu \mathrm{SR}$ signal) is written as $a_{0} P_{Z}(t)$, where $a_{0}$ is the initial asymmetry (a characteristic of the spectrometer and the geometry of the sample) and $P_{Z}(t)$ is the longitudinal muon polarization function which contains information on the local magnetic fields in the sample. In addition, three measurements were done with the transverse field (TF) geometry where $\mathbf{B}_{\text {ext }}$ is perpendicular to $Z$. 


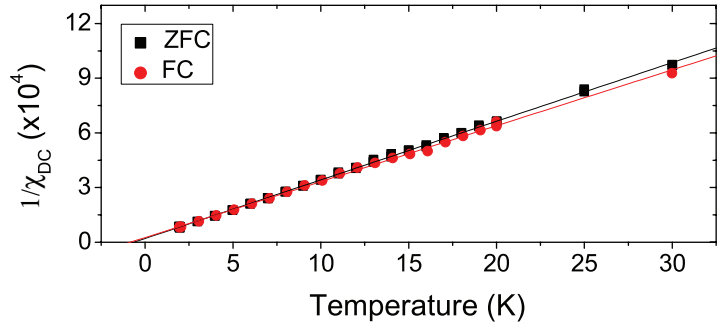

FIG. 1. (Color online) Inverse susceptibility in SI units as a function of temperature in zero field cooled (ZFC) and field cooled (FC) protocols. It was verified that the applied field of $300 \mathrm{mT}$ was small enough to be in the linear regime. We fit the data to $\chi_{\mathrm{DC}}=C_{\mathrm{CW}} /\left(T-T_{\mathrm{CW}}\right)$ and obtain $C_{\mathrm{CW}}=2.45(5) \times 10^{-4} \mathrm{~K}$ and $T_{\mathrm{CW}}=-0.59(2) \mathrm{K}$. Assuming that the magnetic moments in the system are equal to $1 \mu_{\mathrm{B}}$, we can estimate their concentration at the yttrium site (see Discussion section) as $y=3 C_{\mathrm{CW}} a^{3} k_{\mathrm{B}} / 16 \mu_{0} \mu_{\mathrm{B}}^{2}$, where $a$ is the lattice constant. This formula gives $y=0.60(1) \%$.

\section{RESULTS}

It is important to know if there is muonium formation in $\mathrm{Y}_{2} \mathrm{Ti}_{2} \mathrm{O}_{7}$, as this entity is known to diffuse coherently at low temperature in some systems. ${ }^{24}$ Typical ZF and LF (50 $\mathrm{mT}$ ) spectra measured at different temperatures are shown in Fig. 2. The initial asymmetry is temperature and field independent with a value $a_{0} \approx 0.25$ typical for the general purpose spectrometer. This is a strong indication that there is no muonium formation in the whole temperature range. As expected for the absence of muonium, no repolarization (a recovery of the missing asymmetry) by the LF is observed. The absence of muonium is further supported by the TF and ZF signals at $2.4 \mathrm{~K}$ (Fig. 3), where the initial asymmetry is found to be the same for the two spectra and the only frequency observed in TF is that of the applied field. This is further supported by Fourier transform of the TF signal shown in the inset of Fig. 3. If an appreciable vacuummuonium fraction was present, we would expect to observe

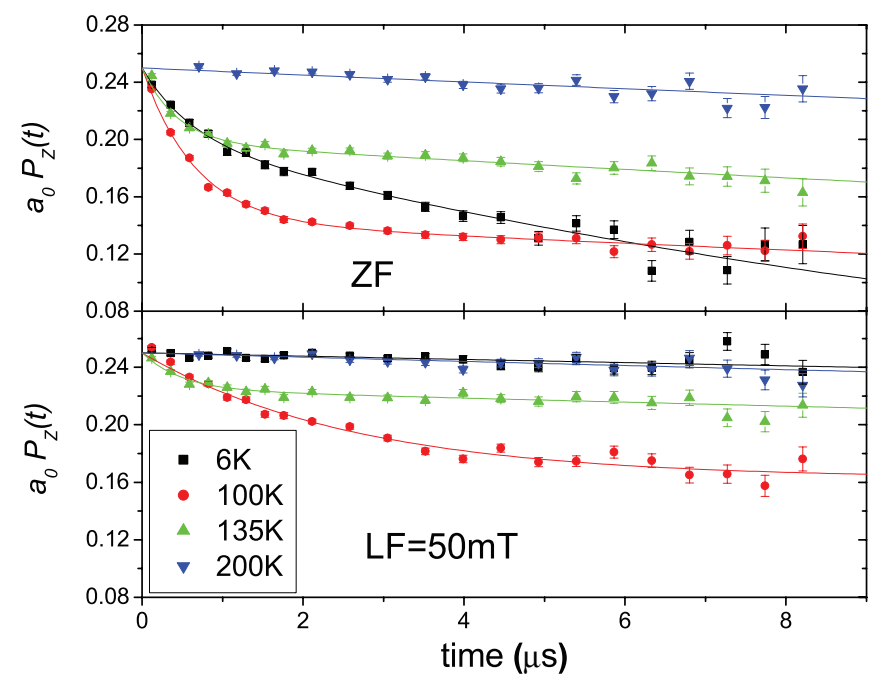

FIG. 2. (Color online) ZF (upper panel) and LF $=50 \mathrm{mT}$ (lower panel) spectra as a function of temperature. The initial muon beam polarization is parallel to the [100] crystal direction. The lines are fits to Eq. (1).

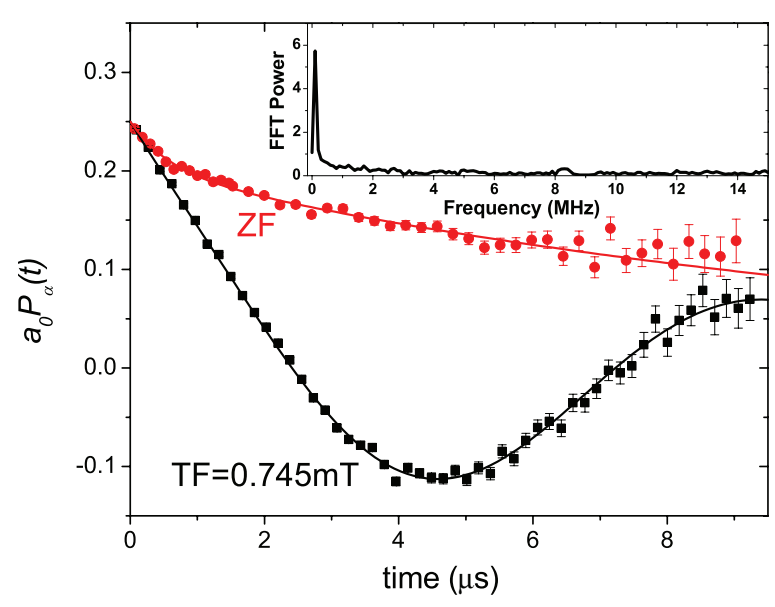

FIG. 3. (Color online) TF $(0.745 \mathrm{mT})$ and $\mathrm{ZF}$ spectra measured at $2.4 \mathrm{~K}$. The inset shows the Fourier transform of the TF spectrum, where a peak is observed at $0.101 \mathrm{MHz}$ corresponding to the applied TF.

an oscillation at a frequency $\approx 103 \gamma_{\mu} B_{\text {ext }} /(2 \pi)=9.8 \mathrm{MHz}$ $\left(\gamma_{\mu}=851.615 \mathrm{Mrad} \mathrm{s}^{-1} \mathrm{~T}^{-1}\right)$ in the Fourier spectrum, ${ }^{24}$ and/or a reduction of the initial asymmetry due to the fast muonium precession. Therefore, we find no evidence of neutral muonium states in $\mathrm{Y}_{2} \mathrm{Ti}_{2} \mathrm{O}_{7}$. The reason for the muonium absence in the pyrochlore oxides is not understood, and it is certainly a subject of much interest.

We turn now to discussing the ZF and LF data in Fig. 2, where we observe a surprisingly high relaxation rate. A priori, electronic magnetism should not be present in $\mathrm{Y}_{2} \mathrm{Ti}_{2} \mathrm{O}_{7}$ since both $\mathrm{Y}^{3+}$ and $\mathrm{Ti}^{4+}$ ions are nonmagnetic. Therefore, only nuclear magnetic moments should be present in this system. However, as shown in Fig. 1, the susceptibility shows a relatively strong paramagnetism. Also, as shown in Fig. 4, not even a field $B_{\text {ext }}=20 \mathrm{mT}$ can completely decouple the relaxation of the $\mu \mathrm{SR}$ signal (usually less than $3 \mathrm{mT}$ are needed to decouple nuclear fields, ${ }^{25}$ which we estimated to be $\approx 20 \mu \mathrm{T}$ in $\mathrm{Y}_{2} \mathrm{Ti}_{2} \mathrm{O}_{7}$ ). Hence we conclude that the relaxation is of electronic origin as proposed by Dunsiger. ${ }^{21}$

\section{DISCUSSION}

To understand the origin of the electronic magnetic moments we first discuss the $\mathrm{Y}_{2} \mathrm{Ti}_{2} \mathrm{O}_{7}$ crystal structure. In general, we note that the ternary pyrochlore compounds are of the general formula $A_{2} B_{2} \mathrm{O}_{7} .{ }^{22}$ The $B$ element can be a transition metal with a variable oxidation state. This gives the possibility of substitution on the $B$ site (see Ref. 26, and references therein). The structure also tolerates vacancies at the $A$ and $O$ sites to a certain extent. Around the $A$ and $B$ cations one finds eight- and sixfold coordination polyhedron of oxygen, respectively. Focusing on $\mathrm{Y}_{2} \mathrm{Ti}_{2} \mathrm{O}_{7}$, in terms of the oxidation states we have $\mathrm{Y}_{2}^{3+} \mathrm{Ti}_{2}^{4+} \mathrm{O}_{7}^{2+}$. $\mathrm{Ti}^{4+}$ is diamagnetic since it is in a $d^{0}$ configuration. Depending on its coordination the $\mathrm{Ti}^{4+}$ ionic radius is 74.4 or $88 \mathrm{pm}$, which is smaller than the $\mathrm{Y}^{3+}$ ionic radius of 104 or $115.9 \mathrm{pm}$. Now we consider a magnetic configuration for at least one of the three involved elements. The obvious candidate is $\mathrm{Ti}^{3+}$, which has about the same ionic radius as $\mathrm{Ti}^{4+}$. This ion is known to be rather 


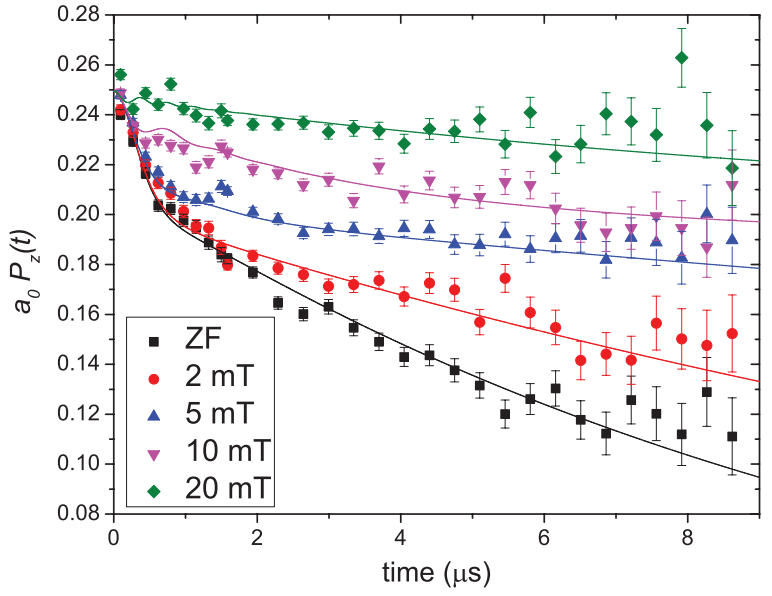

FIG. 4. (Color online) Field dependence of the LF data at $2.4 \mathrm{~K}$. The lines are fits to Eq. (2).

unstable ${ }^{27}$ and it has been suggested to locate it in the $A$ site. ${ }^{22}$ In this case we can write $\left(\mathrm{Y}_{2-x} \mathrm{Ti}_{x}\right) \mathrm{Ti}_{2} \mathrm{O}_{7}$, where we have neglected the substitution of $\mathrm{Y}^{3+}$ in the $B$. This does not change our conclusions since $\mathrm{Y}^{3+}$ is nonmagnetic. ${ }^{28,29} \mathrm{Ti}^{3+}$ has an electronic configuration $d^{1}$ and therefore, as a free ion, carries one Bohr magneton. Another possibility, which is less likely though cannot be ruled out, is $\mathrm{O}$ vacancies in the structure. Such defects will also introduce two $\mathrm{Ti}^{3+}$ magnetic moments for each vacancy. ${ }^{28}$ Note that both types of defects will produce essentially the same effect on the implanted muons and cannot be distinguished in our measurements.

We now consider qualitatively the ZF and $50 \mathrm{mT}$ LF spectra of Fig. 2. In ZF and low temperatures we observe a signal with a slow and a fast relaxing components. As the temperature is increased, the relaxation rate of the fast component decreases slightly and then increases until this component disappears above $150 \mathrm{~K}$. The relaxation of the slow component decreases continuously with temperature. In the LF measurements the overall relaxation rate starts increasing as the temperature is increased, peaks at $100 \mathrm{~K}$, and then it decreases to a small value above $150 \mathrm{~K}$. Referring to the original work of Borghini et al. ${ }^{30,31}$ and the latter works reviewed in Ref. 25, the temperature dependence of the spectra is typical for a muon trapping/detrapping effect. At low temperature the overall relaxation is small because a big fraction of muons are implanted "far" from $\mathrm{Ti}^{3+}$ magnetic defects. As the temperature is increased, the relaxation increases because muons diffuse towards magnetic defects and become trapped. At even higher temperatures, trapping/detrapping become faster leading to a decrease of the relaxation rate due to the fast fluctuating magnetic field sensed by the muons. The $50 \mathrm{mT}$ field is sufficient to quench the relaxation except around $100 \mathrm{~K}$, where a $B_{\text {ext }}=200 \mathrm{mT}$ was needed to suppress the relaxation at this temperature. This is consistent with muons diffusing and accumulating near magnetic defects at this temperature, i.e., the trapping rate is higher than the detrapping rate.

We base our analysis on a multistate model and, for simplicity, we restrict ourselves to a two-state model. ${ }^{30,31} \mathrm{In}$ this model the muon can be diffusing in the undisturbed regions or trapped near to a magnetic impurity. The relaxation rate from muons in the trapped state is expected to be higher since

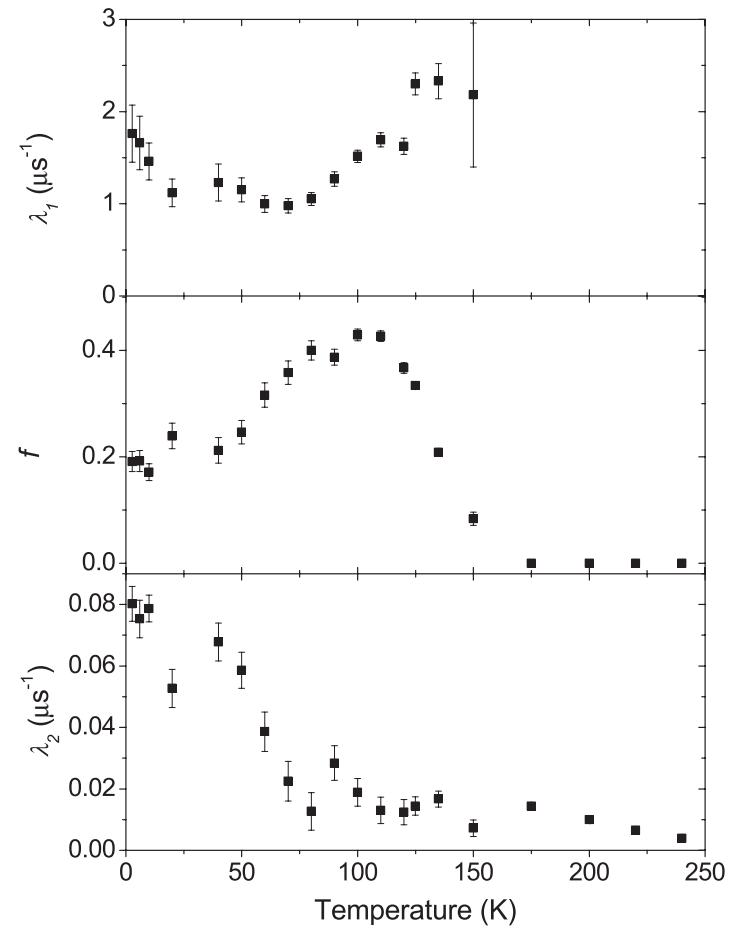

FIG. 5. Temperature dependence of $\lambda_{1}, \lambda_{2}$, and $f$ obtained from fits of the ZF spectra to Eq. (1). $f$ was set to zero for $T>150 \mathrm{~K}$.

they are closer to magnetic defects and therefore experience stronger magnetic fields. Also, muons in undisturbed regions can diffuse and reach trapping sites (at a given trapping rate), while trapped muons can escape traps if the temperature is high enough (at a given detrapping rate). The muon polarization function for such a two-state model can be approximated by the sum of two relaxing signals,

$$
P_{Z}(t)=f \exp \left(-\lambda_{1} t\right)+(1-f) \exp \left(-\lambda_{2} t\right),
$$

where $\lambda_{1}$ and $\lambda_{2}$ are the relaxation rates of the two components and $f$ is the contribution of the first component to the full signal. At the limit of zero trapping/detrapping rates, $\lambda_{1}$ and $\lambda_{2}$ represent the relaxation rate of muons in traps and undisturbed regions, respectively, while $f$ is the fraction of muons in traps. Equation (1) provides a good qualitative description of the ZF data at all temperatures as shown by the solid lines in the top panel of Fig. 2. The temperature dependence of the three parameters in ZF is shown in Fig. 5. We find $f \simeq 0.20$ and temperature independent below $40 \mathrm{~K}$, indicating that the muon trapping and detrapping rates are constant below $40 \mathrm{~K}$. Furthermore, the fact that for these temperatures the signal can be decoupled by small applied fields is evidence that most of the muons cannot diffuse to reach a high field trapping site during the experimental time window $(8.5 \mu \mathrm{s})$. Therefore, we conclude that below $40 \mathrm{~K}$ the trapping and detrapping rates must be small. At higher temperatures $f$ increases due to the enhanced muon trapping caused by a faster diffusion in the undisturbed regions, and then goes to zero at $135 \mathrm{~K}$ indicating that muons trap and detrap so fast that they experience fast fluctuating magnetic fields (motional narrowing) and relax following a single exponential behavior. ${ }^{31}$ We want to point out that fitting the data with a temperature independent $f$ does not 
produce satisfactory fits, and fitting with a power-exponential function produces very bad results for temperatures above $70 \mathrm{~K}$. Also, an implementation of the two state model as presented in Ref. 31 cannot account for the observed temperature dependence of $f$, probably because of the assumption that the relaxation rate within each region (state) is exponential in the whole temperature range.

To study the dynamic behavior at $2.4 \mathrm{~K}$, we fit the LF data using the polarization function (see Fig. 4),

$$
P_{Z}(t)=f P^{\mathrm{KT}}\left(\Delta_{1}, v_{1}, B_{\mathrm{ext}}, t\right)+(1-f) P^{\mathrm{KT}}\left(\Delta_{2}, v_{2}, B_{\mathrm{ext}}, t\right),
$$

where $P^{\mathrm{KT}}$ is the analytical approximation of a Gaussian Kubo-Toyabe function proposed by Keren. ${ }^{32}$ By construction, this equation assumes two independent muon fractions and therefore it represents the two-state model in the limit of zero trapping and detrapping rates. In each region (or state) the muons sense random fields from a Gaussian distribution of width $\Delta_{i}$. This field can fluctuate at a rate $v_{i}$ due to fluctuations of the magnetic defects or hopping of the muon from one site to another within the same region. In the fit, $f$ was fixed to its $\mathrm{ZF}$ value $(0.2)$ and $B_{\text {ext }}$ to the applied LF. The fit is good and completely captures the decoupling of the signal, further supporting our assumption of small muon trapping and detrapping rates. The values of the fitted parameters are: $\Delta_{1}=3.00(5) \mathrm{mT}, v_{1}=2.4(2) \mu \mathrm{s}^{-1}, \Delta_{2}=0.318(5) \mathrm{mT}$, and $\nu_{2}=1.52(7) \mu \mathrm{s}^{-1} . \Delta_{1}$, which corresponds to the trapped state, is consistent with the dipolar field expected in a region of $8 \AA$ around a $1 \mu_{B}$ magnetic impurity (see discussion below), and $v_{2}$ imposes a maximum limit in the hopping rate of muons in the undisturbed regions. We want to note that $v_{1}$ and $v_{2}$ are very similar and, in fact, the data can be fit with a common fluctuation rate with no significant effect on the quality of the fit. Since the contribution from the paramagnetic defects to $v_{1}$ and $v_{2}$ is the same, this indicates that the hopping rate in the undisturbed regions is probably much smaller than $v_{2}$.

One point that needs to be discussed is the relatively large $f$ fraction found at low temperature. There are sixteen $\mathrm{Y}^{3+}$ ions per cubic unit cell of volume $V_{\mathrm{cc}}=a^{3}$. Let us denote $y$ the percentage of magnetic defects relative to the $\mathrm{Y}^{3+}$ population: $y=x / 2$, i.e., there are $16 y=8 x \mathrm{Ti}^{3+}$ ions in $V_{\text {cc }}$. Let us assume that a muon is trapped in a domain of relative weight $f$ when implanted within a distance $d$ from a defect, and also that the size and number of these domains are small enough such that they do not overlap. Therefore, the volume around a defect in which a muon is trapped is $V_{\mu}=8 x(4 \pi / 3) d^{3}$. Since $f=$ $V_{\mu} / V_{\text {cc }}$, we conclude that $d=[(3 / 32 \pi)(f / x)]^{1 / 3} a$. With $f \simeq$ 0.20 and $y=0.6 \%$ (see caption of Fig. 1), we compute $d=$ $0.79 a=8 \AA$, which is a reasonable value. ${ }^{33}$ This relatively long-range influence of a defect is due to the long range of both the dipolar interaction and the nature of the elastic distortion field. ${ }^{34,35}$

\section{SUMMARY AND CONCLUSIONS}

In conclusion, we find that the muon hopping rate at $2.4 \mathrm{~K}$, in both stopping regions, is smaller than $\approx 2 \mu \mathrm{s}^{-1}$. This value is far below that observed for coherent diffusion of positive muons in metals as well as the $\sim 10^{3} \mu \mathrm{s}^{-1}$ proposed for $\mathrm{Dy}_{2} \mathrm{Ti}_{2} \mathrm{O}_{7} \cdot{ }^{20}$ Therefore, we find it unlikely that coherent muon diffusion is present in $\mathrm{Y}_{2} \mathrm{Ti}_{2} \mathrm{O}_{7}$. Nevertheless, an appropriate theoretical microscopic calculation for the behavior of positive muons in this system is needed to completely rule out or confirm coherent muon diffusion. However, any theory should include the extended magnetic defects found by our measurements. In this regard, our observations add to the growing evidence that defects in pyrochlores are important to understand their magnetic properties, ${ }^{13,28,36-40}$ and in particular those of $\mathrm{Dy}_{2} \mathrm{Ti}_{2} \mathrm{O}_{7} \cdot{ }^{29}$ Finally, an analysis to extract trapping and detrapping rates in the whole temperature range would have to follow the lines of that in Ref. 31 but allow for nonexponential relaxation rates and/or going beyond the two states model.

\section{ACKNOWLEDGMENTS}

We would like to thank Marisa Medarde for her support in the magnetization measurements and A. Amato for the $\mu \mathrm{SR}$ assistance.
*Corresponding author: joroal@hotmail.com

†Corresponding author: zaher.salman@psi.ch

${ }^{1}$ J. S. Gardner, M. J. P. Gingras, and J. E. Greedan, Rev. Mod. Phys. 82, 53 (2010).

${ }^{2}$ M. J. Harris, S. T. Bramwell, D. F. McMorrow, T. Zeiske, and K. W. Godfrey, Phys. Rev. Lett. 79, 2554 (1997).

${ }^{3}$ A. P. Ramirez, A. Hayashi, R. J. Cava, R. Siddharthan, and B. S. Shastry, Nature (London) 399, 333 (1999).

${ }^{4}$ S. R. Dunsiger, A. A. Aczel, C. Arguello, H. Dabkowska, A. Dabkowski, M.-H. Du, T. Goko, B. Javanparast, T. Lin, F. L. Ning et al., Phys. Rev. Lett. 107, 207207 (2011).

${ }^{5}$ J. A. Hodges, P. Bonville, A. Forget, A. Yaouanc, P. Dalmas de Réotier, G. André, M. Rams, K. Królas, C. Ritter, P. C. M. Gubbens et al., Phys. Rev. Lett. 88, 077204 (2002).

${ }^{6}$ I. Mirebeau, A. Apetrei, J. Rodríguez-Carvajal, P. Bonville, A. Forget, D. Colson, V. Glazkov, J. P. Sanchez, O. Isnard, and E. Suard, Phys. Rev. Lett. 94, 246402 (2005).
${ }^{7}$ P. Dalmas de Réotier, A. Yaouanc, L. Keller, A. Cervellino, B. Roessli, C. Baines, A. Forget, C. Vaju, P. C. M. Gubbens, A. Amato et al., Phys. Rev. Lett. 96, 127202 (2006).

${ }^{8}$ E. Bertin, P. Bonville, J.-P. Bouchaud, J. A. Hodges, J. P. Sanchez, and P. Vulliet, Eur. Phys. J. B 27, 347 (2002).

${ }^{9}$ A. Yaouanc, P. Dalmas de Réotier, V. Glazkov, C. Marin, P. Bonville, J. A. Hodges, P. C. M. Gubbens, S. Sakarya, and C. Baines, Phys. Rev. Lett. 95, 047203 (2005).

${ }^{10}$ Y. Chapuis, P. Dalmas de Réotier, C. Marin, A. Yaouanc, A. Forget, A. Amato, and C. Baines, Physica B 404, 686 (2009).

${ }^{11}$ J. Lago, T. Lancaster, S. J. Blundell, S. T. Bramwell, F. L. Pratt, M. Shirai, and C. Baines, J. Phys.: Condens. Matter 17, 979 (2005).

${ }^{12}$ P. Dalmas de Réotier, A. Yaouanc, Y. Chapuis, S. H. Curnoe, B. Grenier, E. Ressouche, C. Marin, J. Lago, C. Baines, and S. R. Giblin, Phys. Rev. B 86, 104424 (2012).

${ }^{13}$ A. Yaouanc, P. Dalmas de Réotier, Y. Chapuis, C. Marin, S. Vanishri, D. Aoki, B. Fåk, L. P. Regnault, C. Buisson, A. Amato et al., Phys. Rev. B 84, 184403 (2011). 
${ }^{14}$ J. S. Gardner, S. R. Dunsiger, B. D. Gaulin, M. J. P. Gingras, J. E. Greedan, R. F. Kiefl, M. D. Lumsden, W. A. MacFarlane, N. P. Raju, J. E. Sonier et al., Phys. Rev. Lett. 82, 1012 (1999).

${ }^{15}$ Y. J. Uemura, A. Keren, K. Kojima, L. P. Le, G. M. Luke, W. D. Wu, Y. Ajiro, T. Asano, Y. Kuriyama, M. Mekata et al., Phys. Rev. Lett. 73, 3306 (1994).

${ }^{16}$ K. C. Rule, G. Ehlers, J. S. Gardner, Y. Qiu, E. Moskvin, K. Kiefer, and S. Gerischer, J. Phys.: Condens. Matter 21, 486005 (2009).

${ }^{17}$ J. Lago, S. J. Blundell, and C. Baines, J. Phys.: Condens. Matter 19, 326210 (2007).

${ }^{18}$ J. S. Gardner, G. Ehlers, P. Fouquet, B. Farago, and J. R. Stewart, J. Phys.: Condens. Matter 23, 164220 (2011).

${ }^{19}$ P. A. McClarty, J. N. Cosman, A. G. D. Maestro, and M. J. P. Gingras, J. Phys.: Condens. Matter 23, 164216 (2011).

${ }^{20}$ P. Quémerais, P. McClarty, and R. Moessner, Phys. Rev. Lett. 109, 127601 (2012).

${ }^{21}$ S. R. Dunsiger, Ph.D. thesis, University of British Columbia, Vancouver, 2000.

${ }^{22}$ M. A. Subramanian, G. Aravamudan, and G. V. S. Rao, Prog. Solid State Chem. 15, 55 (1983).

${ }^{23}$ A. Schenck and F. N. Gygax, in Handbook of Magnetic Materials, edited by K. H. J. Buschow (Elsevier, Amsterdam, 1995), Vol. 9.

${ }^{24}$ A. Yaouanc and P. Dalmas de Réotier, Muon Spin Rotation, Relaxation, and Resonance: Applications to Condensed Matter, International Series of Monographs on Physics Vol. 147 (Oxford University Press, Oxford, 2011).

${ }^{25}$ E. B. Karlsson, Solid State Phenomena As Seen by Muons, Protons, and Excited Nuclei (Clarendon, Oxford, 1995).

${ }^{26}$ B. G. Ueland, J. S. Gardner, A. J. Williams, M. L. Dahlberg, J. G. Kim, Y. Qiu, J. R. D. Copley, P. Schiffer, and R. J. Cava, Phys. Rev. B 81, 060408 (2010).
${ }^{27}$ A. Abragam and B. Bleaney, Electron Paramagnetic Resonance of Transition Ions (Clarendon, Oxford, 1970).

${ }^{28}$ K. A. Ross, T. Proffen, H. A. Dabkowska, J. A. Quilliam, L. R. Yaraskavitch, J. B. Kycia, and B. D. Gaulin, Phys. Rev. B 86, 174424 (2012).

${ }^{29}$ H. M. Revell, L. R. Yaraskavitch, J. D. Mason, K. A. Ross, H. M. L. Noad, H. A. Dabkowska, B. D. Gaulin, P. Henelius, and J. B. Kycia, Nature Phys. 9, 34 (2013).

${ }^{30}$ M. Borghini, T. O. Niinikoski, J. C. Soulié, O. Hartmann, E. Karlsson, L. O. Norlin, K. Pernestål, K. W. Kehr, D. Richter, and E. Walker, Phys. Rev. Lett. 40, 1723 (1978).

${ }^{31}$ K. W. Kehr, G. Honig, and D. Richter, Z. Phys. B 32, 49 (1978).

${ }^{32}$ A. Keren, Phys. Rev. B 50, 10039 (1994).

${ }^{33}$ S. Sanna, G. Allodi, G. Concas, A. D. Hillier, and R. De Renzi, Phys. Rev. Lett. 93, 207001 (2004).

${ }^{34}$ H. J. D. Eshelby, in Solid State Physics, edited by F. Seitz and D. Turnbull (Academic Press, New York, 1956), Vol. 3, p. 79.

${ }^{35} \mathrm{G}$. Leibfried and N. Breuer, Point Defects in Metals I. Introduction to the Theory, Springer Tracts in Modern Physics Vol. 81 (Springer, Berlin, 1978).

${ }^{36}$ Y. Chapuis, Ph.D. thesis, Université Joseph Fourier, Grenoble, 2009.

${ }^{37}$ Y. Chapuis, A. Yaouanc, P. Dalmas de Réotier, C. Marin, S. Vanishri, S. H. Curnoe, C. Vâju, and A. Forget, Phys. Rev. B 82, 100402(R) (2010).

${ }^{38}$ H. Takatsu, H. Kadowaki, T. J. Sato, J. W. Lynn, Y. Tabata, T. Yamazaki, and K. Matsuhira, J. Phys.: Condens. Matter 24, 052201 (2012)

${ }^{39}$ A. Yaouanc, P. Dalmas de Réotier, C. Marin, and V. Glazkov, Phys. Rev. B 84, 172408 (2011).

${ }^{40}$ K. A. Ross, L. R. Yaraskavitch, M. Laver, J. S. Gardner, J. A. Quilliam, S. Meng, J. B. Kycia, D. K. Singh, T. Proffen, H. A. Dabkowska et al., Phys. Rev. B 84, 174442 (2011). 